Numerical simulation of circulating tumor cell separation in a dielectrophoresis based $Y-Y$ shaped microfluidic device

Xiangzhi Zhang, Xiawei Xu, Yong Ren, Yuying Yan, Aiguo Wu

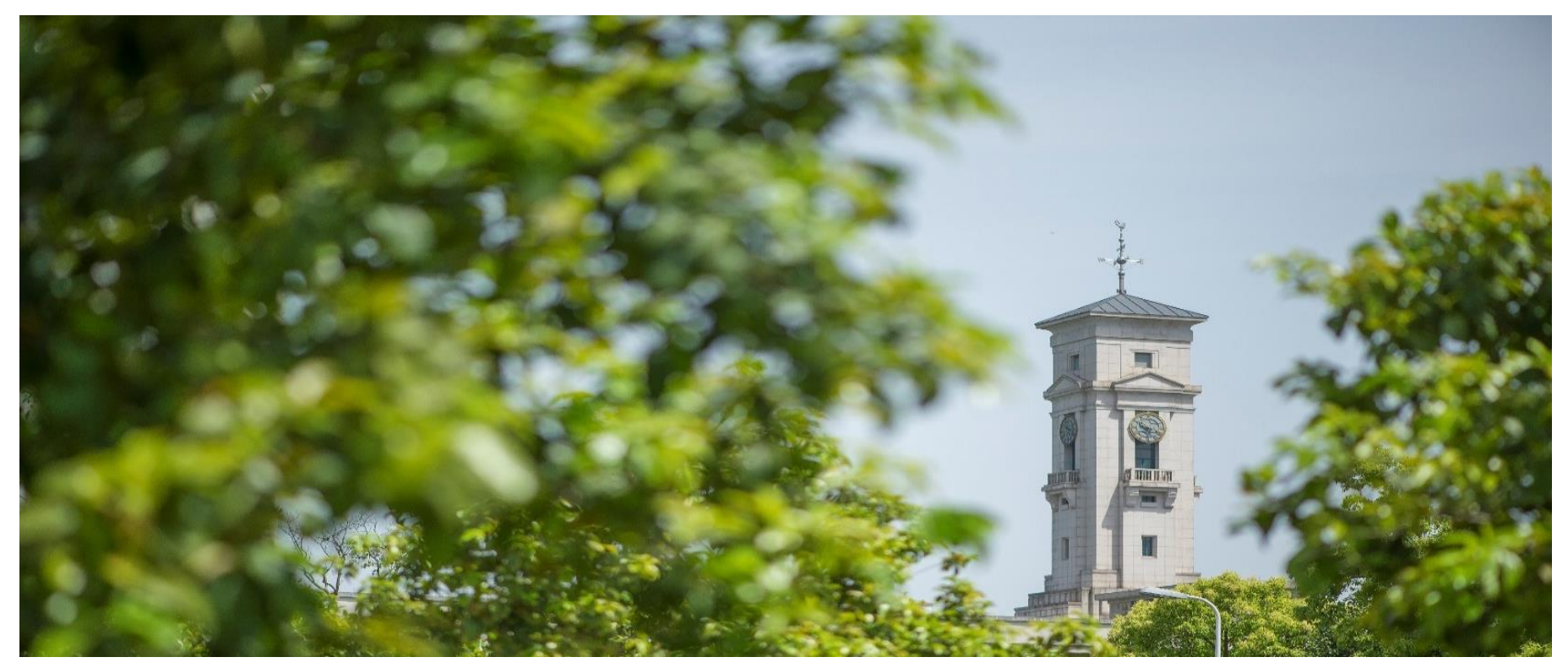


University of Nottingham Ningbo China, 199 Taikang East Road, Ningbo, 315100, Zhejiang, China.

First published 2020

This work is made available under the terms of the Creative Commons Attribution 4.0 International License:

http://creativecommons.org/licenses/by/4.0

The work is licenced to the University of Nottingham Ningbo China under the Global University Publication Licence:

https://www.nottingham.edu.cn/en/library/documents/researchsupport/global-university-publications-licence-2.0.pdf 


\title{
Numerical simulation of circulating tumor cell separation in a dielectrophoresis based Y-Y shaped microfluidic device
}

\author{
Xiangzhi Zhang ${ }^{1}$, Xiawei $\mathrm{Xu}^{1,2,3}$, Yong Ren ${ }^{1,3^{*}}$, Yuying Yan ${ }^{1,4^{*}}$, Aiguo $\mathrm{Wu}^{2^{*}}$ \\ ${ }^{1}$ Research Group for Fluids and Thermal Engineering, University of Nottingham Ningbo
} China, Ningbo, China

${ }^{2}$ Cixi Institute of Biomedical Engineering, CAS Key Laboratory of Magnetic Materials and Devices, \& Key Laboratory of Additive Manufacturing Materials of Zhejiang Province, Ningbo Institute of Materials Technology and Engineering, Chinese Academy of Sciences, Ningbo, China

${ }^{3}$ Department of Mechanical, Materials and Manufacturing Engineering, University of Nottingham Ningbo China, Ningbo, China

${ }^{4}$ Research Group for Fluids and Thermal Engineering, University of Nottingham, Nottingham, UK

\section{${ }^{*}$ Correspondence Email:}

yong.ren@nottingham.edu.cn; yuying.yan@nottingham.ac.uk; aiguo@nimte.ac.cn

\begin{abstract}
Efficient separation of circulating tumor cells from biological samples to promote early diagnosis of cancer is important but challenging, especially for non-small lung cancer. In this article, a Y-Y shaped microfluidic device was designed to isolate non-small lung cells with dielectrophoresis approach. Numerical simulations were conducted that the trajectories of cells were traced by solving the electric potential distribution and the flow in channel. The effects of inlet velocity ratio of blood sample and buffer on separation performance were studied and optimized by conducting numerical simulations. Under optimal operating conditions, the separation efficiency can reach around 99\%, which is achieved with $100 \mathrm{kHz} \mathrm{AC}$, electrodes potential of $1.6 \mathrm{~V}$ to $2.2 \mathrm{~V}$, and flowrate ratio of 1.9 to 2.5 . This study presents a potential route for an efficient, relatively simple and low-cost method for circulating tumor cells separation.
\end{abstract}

Keywords: circulating tumor cells, separation, dielectrophoresis 


\section{Introduction}

Circulating tumor cells (CTCs) are tumor cells shedding from the solid tumor, entering into the bloodstream, surviving in the circulating system, and traveling to distant organs, which resulting in metastasis. The spread of CTCs from initial sites to form distant secondary tumors is one of the main cause of death in cancer patients.[1] CTC detection has received enormous attention because of the potential value in the early diagnosis of cancer, prediction of clinical prognosis, evaluation of therapeutic efficiency, development of targeted drugs and personalized medicine of malignant cancer. Since the concentration of CTCs in the blood is extremely low, on the order of one out of a billion cells, CTCs must be isolated to achieve a high concentration in order to facilitate liquid biopsy efficiently.[2] At present, the technologies commonly used in CTC separation can be categorized as CellSearch system, Nano Velcro CTC chip, microfluidic technology, and other physical separation methods including microfilter and density gradient centrifugation.[3] These methods are classified into positive isolation focusing on CTC capture and negative isolation focusing on capture of leukocytes or erythrocyte. CellSearch system is based on immunomagnetic method which uses magnetic nanoparticles coated with antibodies, aptamers or peptides to capture CTCs and isolate them from blood cells with the impacts of a magnetic field.[4] Although its false negative rate is high and consumes large amounts of blood samples, the standardization of CellSearch makes it the only CTC detection method currently approved by FDA. Nano Velcro CTC chip has a large surface area, which increases the contacting area with CTCs and thus improving the isolation efficiency, while it requires excessive consumption of antibodies.[5] Microfluidic technologies are classified into label-dependent isolation and label-independent isolation. The advantages of Label- 
independent isolation methods include simple operation process, keeping cell integrity and no need for antibodies. Label-dependent isolation methods commonly use Epithelial cell adhesion molecule (EpCAM) to target CTCs which can improve the specificity of methods.[6] Saliba et al.[7] developed a microfluidic device with antibody functionalized superparamagnetic beads that can self-assemble with magnetic traps in the microchannel. The capturing efficiency can reach higher than $94 \%$, yet the self-assembled magnetic columns used in these methods are often unstable due to the large hydrodynamic drag force, resulting in low throughput. A magnetic force gradient based microfluidic chip was developed to separate CTCs depending on their expression level of EpCAM.[8] This method is of prognostic value in patients with solid tumors, such as advanced breast, colon, and prostate cancer, while its poor sensitivity for non-small lung cancer (NSCLC) restricts the utilization in treatment.[9] Physical separation methods such as morphology-based microfilter, and density gradient centrifugation methods have advantages of simple operation, maintaining cell integrity and no need for expensive antibodies. However, such methods are limited by the low specificity and high false positive. Among negative isolation methods, magnetic nanobeads coated with anti-CD45 antibodies have been commonly used for separating leukocytes from blood samples, thereby enriching CTCs.[10] Although the recovery rate of this negative method is higher than that of positive method, the purity of isolated CTCs is relatively lower; therefore, further isolation is required.

Dielectrophoresis (DEP) microfluidic technologies have been intensively investigated for CTC separation and detection. DEP is one of the most common electrokinetic phenomena and was first described by Pohl.[11] When a nonuniform electric field is applied, it will induce a dipole moment on the particle due to the electrical polarization at the particle's 
surface. Driven by dielectrophoretic force, the particle has to be migrated in the nonuniform electric field to achieve electrostatic equilibrium. The magnitude of dielectrophoretic force (DEP force) is proportional to the gradient of electric potential, and also depends on the size, deformation and dielectric constant of substance. DEP separations of cells are sensitive to the sizes, shapes, and dielectric properties of the particles, which allows this method to be used widely in bioengineering. In the low frequency region with frequency roughly lower than $10 \mathrm{MHz}$, the DEP behavior of a cell is largely determined by extracellular factors, including membrane-bound protein, cell size, solution conductivity, and electric permittivity.[12] Becker et al.[13] developed a method to isolate viable cultured breast tumor cells from peripheral blood using DEP method, and succeeded in isolating cultured leukemia cells from blood and cultured breast tumor cells from CD341 hemopoietic stem cells.[14] DEP techniques have been evaluated by scholars to manipulate and sort tumor cells.[15][16][17][18] However, previous studies used small electrode arrays, limiting the number of cells that can be processed to a maximum of hundreds of thousands. During DEP isolation process, eluate flow rate, and cell loading concentration influenced the efficiency of DEP isolation. The effects of these factors were analyzed and theoretical models were introduced to optimize the design of DEP-based cell separators.[19] Despite of the rapid development of microfluidics-based CTC separation technology, it remains challenging to develop CTC isolation technologies with improved sensibility, specificity and efficiency without compromising the integrity and activity of isolated CTCs. Besides, the dependence of dielectrophoretic force on the cell size makes cell separation with little difference of dielectric properties a challenge. 


\section{Approach}

\subsection{Magnitude of DEP force}

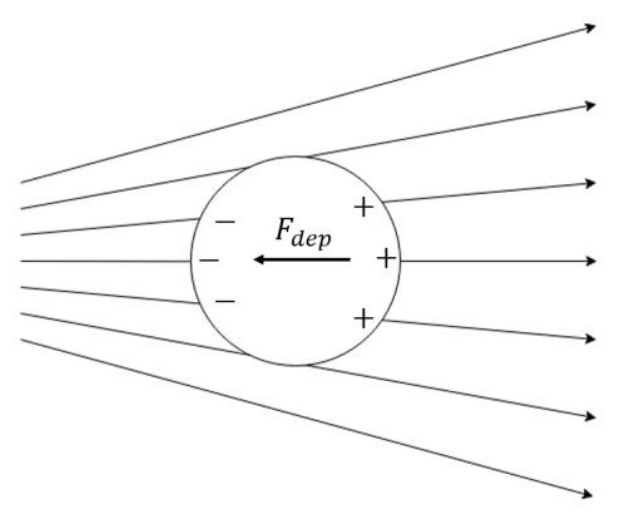

Figure 1. Sketch of DEP force. The DEP force is the interaction of a nonuniform electric field with the dipole moment it induces.

Primarily, DEP rises from the non-linear interaction applied on an extended dipole body subjected to a nonuniform electric field and surrounded by a dielectric medium, which can be harnessed to move and manipulate microparticles that suspended in liquid media.[20] Correspondingly, the DEP force is the interaction of a nonuniform electric field with the dipole moment it induces (shown in Fig. 1), the magnitude of which is given by[21]

$$
F_{\text {dep }}=2 \pi r_{p}^{3} \epsilon_{0} \operatorname{Re}\left(\epsilon_{r, f}^{*}\right) \operatorname{Re}\left(K_{C M}\right) \nabla|\bar{E}|^{2}
$$

where $r_{p}$ is the particle radius, $\epsilon_{0}=8.854187817 \times 10^{-12} \mathrm{~F} / \mathrm{m}$ is the permittivity of vacuum, $\epsilon_{r, f}^{*}$ is the complex relative permittivity of fluid and $\operatorname{Re}\left(\epsilon_{r, f}^{*}\right)$ denotes its real part, $K_{C M}$ is the Clausius-Mossotti factor and $\operatorname{Re}\left(K_{C M}\right)$ denotes its real part, and $\bar{E}$ denotes the root mean square electric field. The Clausius-Mossotti factor is a parameter to help describe the dielectric property, defined as

$$
K_{C M}=\frac{\epsilon_{r, p}^{*}-\epsilon_{r, f}^{*}}{\epsilon_{r, p}^{*}+2 \epsilon_{r, f}^{*}}
$$


where $\epsilon_{r, p}^{*}$ is the complex relative permittivity of a specific particle.

The complex permittivity of phase $i$ is expressed as

$$
\epsilon_{r}^{*}=\epsilon_{r}-\frac{i \sigma}{\omega \epsilon_{0}}
$$

where $\epsilon_{r}$ denotes the relative permittivity, $\sigma$ denotes the electrical conductivity, and $\omega$ is the angular frequency of the electric field.

\subsection{Problem description}

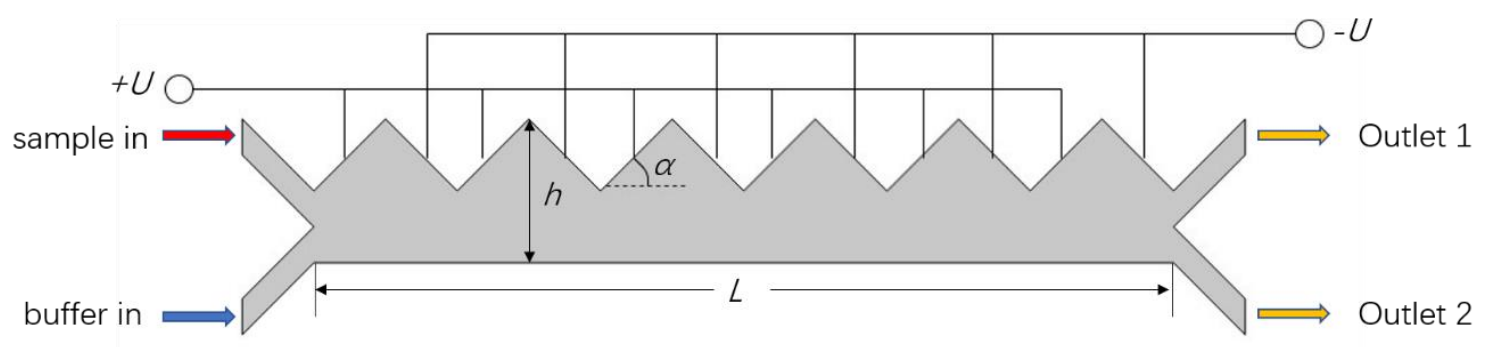

Figure 2. The configuration of the DEP-microchannel cell sorting system, the width of the microchannel $h=200 \mu m$, the length of channel $L=1200 \mu m$, the angle $\alpha=45^{\circ}$. Previous research has shown that NSCLC are relatively insensitive to some microfluidic isolation methods, which makes their isolation operation difficult.[22] Based on dielectric differences, DEP has great potential to identify and isolate CTCs from other blood cells. In this study, a DEP-assisted microfluidic device is presented and adopted in the isolation of 5 types of NSCLC (HOP-62, HOP-92, NCI-H226, NCI-H23, and EKVX) from blood samples.

As shown in Fig. 2, the cell sorting system consists of low-voltage electrodes and a Y-Y configured microchannel, which is connected with two inlets and two outlets. (the width of inlets and outlets $w=50 \mu m$ ) The upper wall of the channel is comprised of successive 
triangles with alternating sides charged oppositely, which generates a nonuniform electric field in flow region. A blood sample containing erythrocytes (the density $\rho=1050 \mathrm{~kg} / \mathrm{m}^{3}$, the diameter $d=5 \mu \mathrm{m}$, the electric conductivity $\sigma=0.31 \mathrm{~S} / \mathrm{m}$, and the relative permittivity $\epsilon_{r}=59$ ) and CTCs (physical properties as Table. 1) is fed into the flow channel from the upper inlet, while a buffer[23] (the density $\rho=1000 \mathrm{~kg} / \mathrm{m}^{3}$, the dynamic viscosity $\mu=0.001 \mathrm{~Pa} \cdot \mathrm{s}$, the electric conductivity $\sigma=0.055 \mathrm{~S} / \mathrm{m}$, and the relative permittivity $\epsilon_{r}=79$ ) is fed from the lower inlet, which is used to concentrate the cells to accumulate along one side of the channel. After entering the channel, the two streams mix at the intersection of the long channel. When the mixed fluid flow through the channel, due to the effect of the nonuniform electric field, the cells will be subjected to DEP forces with different magnitudes, which are proportional to the sizes of the cells. Since the sizes of CTCs are larger than that of erythrocytes, CTCs will be repelled selectively and captured at the Outlet 2, while other cells will escape from the Outlet 1, thus achieving a cell separation process.

\subsection{Dielectric properties of CTCS}

Gascoyne et al.[24] measured the dielectric properties of NCI-60 panel of tumor cell types and obtained the correlations between the dielectric properties and exterior morphology. According to their study, there exists a correlation for a spherical mammalian cell when DEP frequency $f<1 M H z$ :

$$
\sigma_{p}=\sqrt{2} \pi r_{p} C_{m e m} f_{c o}
$$

where $\sigma_{p}$ is the medium conductivity, $C_{m e m}$ is the capacitance per unit area of the cell plasma membrane, and $f_{c o}$ is the cell crossover frequency, which is proportional to the rate 
at which the plasma membrane capacitance can be charged in the ionic milieu of the suspending medium in response to an applied electric field. The parameters about CTC properties acquired by them are adopted in this study to conduct numerical simulation in this study.[24]

Table. 1 Dielectric properties of CTCs.

\begin{tabular}{ccccc}
\hline Cell line & $r_{p} / \mu m$ & $f_{c o} / k H z$ & $C_{m e m} / m F \cdot m^{-2}$ & $\sigma_{p} / S \cdot m^{-1}$ \\
\hline HOP-62 & 9.84 & 33 & 20.7 & 0.029844 \\
HOP-92 & 10.68 & 30.8 & 20.5 & 0.02994 \\
NCI-H226 & 8.57 & 23.4 & 33.6 & 0.029917 \\
NCI-H23 & 8.94 & 59.1 & 12.8 & 0.030027 \\
EKVX & 10.74 & 30.4 & 20.6 & 0.029862 \\
\hline
\end{tabular}

\subsection{Governing equations and boundary conditions}

In this study, the numerical model is comprised with an electric field and flow field. According to Eq. (1), the DEP force is generated due to a nonuniform electric field, the intensity of which is given by

$$
E=-\nabla U
$$

where $U$ represents the electric potential. The electric field distribution is induced by the electric potential of the upper wall, where there is $U= \pm U_{0}\left(U_{0}\right.$ is the electric potential exerted on the electrodes).

The flow of fluids in channel is governed by Navier-Stokes equations:

$$
\frac{\partial u}{\partial x}+\frac{\partial v}{\partial y}=0
$$




$$
\begin{aligned}
& u \frac{\partial u}{\partial x}+v \frac{\partial u}{\partial y}=-\frac{1}{\rho} \frac{\partial p}{\partial x}+\frac{\mu}{\rho}\left(\frac{\partial^{2} u}{\partial x^{2}}+\frac{\partial^{2} u}{\partial y}\right) \\
& u \frac{\partial v}{\partial x}+v \frac{\partial v}{\partial y}=-\frac{1}{\rho} \frac{\partial p}{\partial y}+\frac{\mu}{\rho}\left(\frac{\partial^{2} v}{\partial y}+\frac{\partial^{2} v}{\partial y^{2}}\right)
\end{aligned}
$$

where $u$ and $v$ are the velocity components of fluid, $\rho$ is the density of fluid, and $\mu$ is the dynamic viscosity of fluid. The boundary conditions of inlets are controlled by the normal inflow velocity of blood $u_{1}$ and the normal inflow velocity of buffer $u_{1}$, while the outlet boundary condition is controlled by the pressure $p_{0}=0$.

The trajectories of cells are traced by solving the motion equation, which is given by impulse theorem

$$
\left\{\begin{array}{l}
\frac{d(m u)}{d t}=F_{x} \\
\frac{d(m v)}{d t}=F_{y}
\end{array}\right.
$$

where $m$ is the mass of a cell; $F_{x}$ and $F_{y}$ are the components of the force source $F$, which consists of three terms:

$$
F=F_{d}+F_{b}+F_{D E P}
$$

where $F_{d}$ is the drag force term, which is proportional to the relative velocity between the cell and the fluid $u_{r}=u_{f}-u_{p}$ :

$$
F_{d}=\frac{18 \mu_{f}}{\rho_{p} d_{p}^{2}} m_{p} u_{r}
$$

where $\mu_{f}$ is the viscosity of fluid, $\rho_{p}$ is the density of particle, and $m_{p}$ is the mass of particle;

$F_{b}$ is the Brownian force term, which depends on the temperature $T$ and the radius of particle $r_{p}$ :

$$
F_{b}=\zeta \sqrt{\frac{12 \pi k_{b} \mu_{f} T r_{p}}{\Delta t}}
$$


where $\zeta$ is a normally distributed random number with a mean of zero and unit standard

deviation, $k_{b}=1.38064852 \times 10^{-23} \mathrm{~J} / K$ is the Boltzmann constant, $\Delta t$ is the time step;[25]

and the third term represents the DEP force, which is proportional to the gradient of the electric field. (referring to Eq. 1)

\section{Results and discussion}

In this study, the separation performance of the DEP-assisted microfluidic method is evaluated by exploring the trajectories of five kinds of NSCLC (HOP-62, HOP-92, NCIH226, NCI-H23, and EKVX) in channel, accompanied by erythrocytes as a control group. The DEP force is induced by a nonuniform electric field, which is generated by electrodes arranged at the wall surface of microchannel on one side, which are connected with power sources. Shown in Fig. 3, the area with high potential are mainly distributed in the triangle regions. The pressure and axial velocity distributions are shown in Fig. 4 and Fig. 5, respectively. These figures indicate the presence of a pulsing accelerating-decelerating flow regime, which will promote the cells in liquid to the triangle regions where the velocity of fluid is relatively lower. 

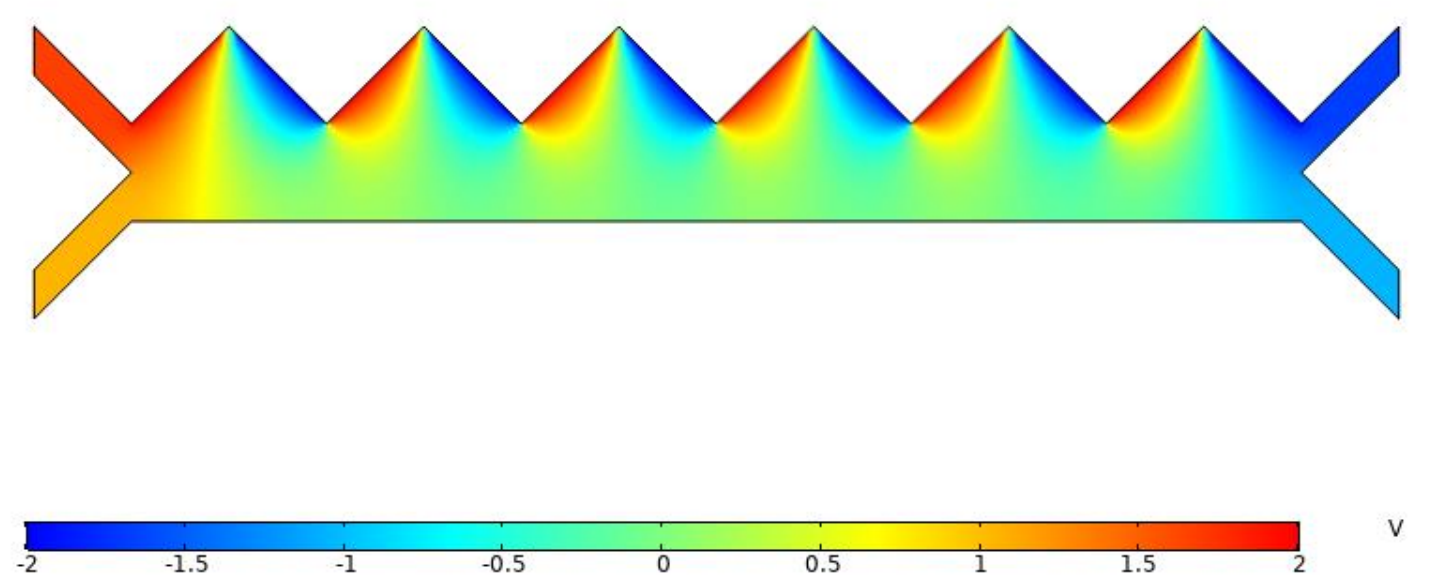

Figure 3. The electric potential distribution in the domain when $f=100 \mathrm{kHz}, U_{0}=2 \mathrm{~V}$.

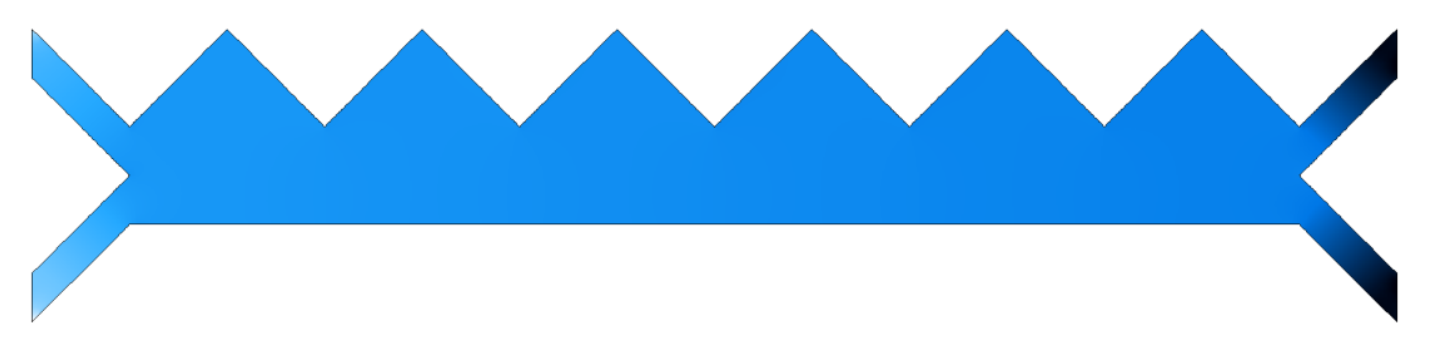

\begin{tabular}{lllllllll}
\hline 0 & 0.5 & 1 & 1.5 & 2 & 2.5 & 3 & 3.5 & $\mathrm{~Pa}$
\end{tabular}

Figure 4 . The pressure distribution when $u_{s}=200 \mu \mathrm{m} / \mathrm{s}, u_{b} /=400 \mu \mathrm{m} / \mathrm{s}$. 

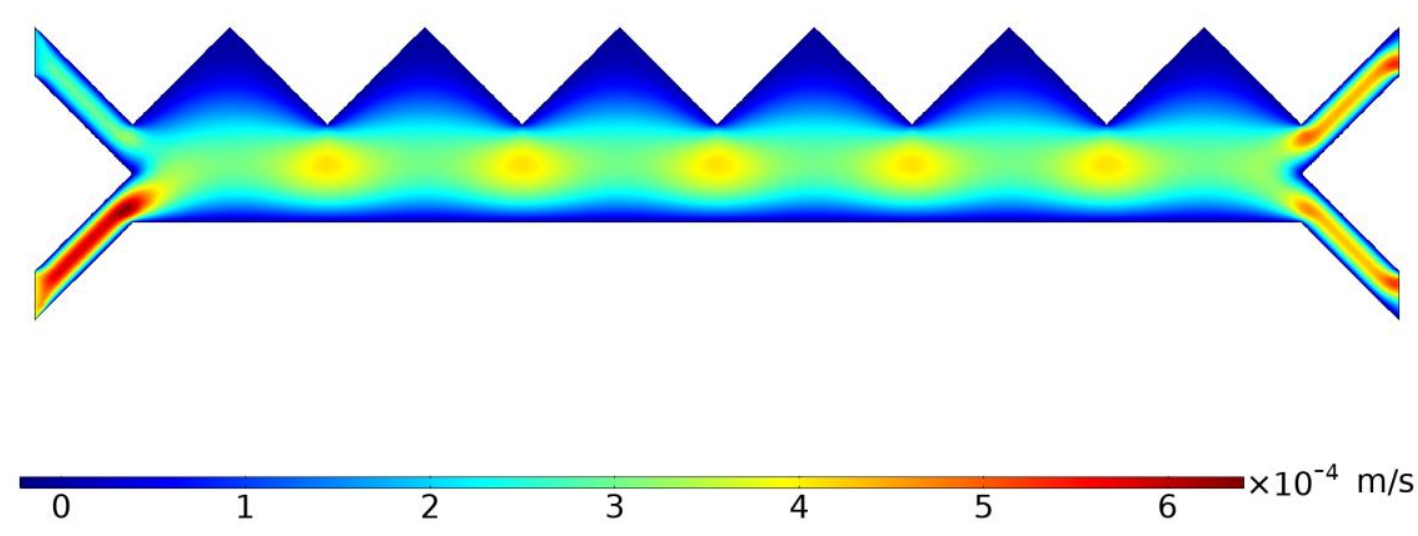

Figure 5. The axial velocity $(\mathrm{m} / \mathrm{s})$ of the fluids when $u_{s}=200 \mu \mathrm{m} / \mathrm{s}, u_{b} /=400 \mu \mathrm{m} / \mathrm{s}$. The trajectories of erythrocytes and CTCs (HOP-62 in Fig. 6) in the channel with or without the impact of DEP force are shown in Fig. 6. According to the figure, it can be seen that all cells share a same trajectory and are collected at Outlet 1without the effect of DEP. In comparison, CTCs are caught at Outlet 1 while erythrocytes escape the channel through the Outlet 2, which proving that the separation is achieved with DEP forces applied on cells.

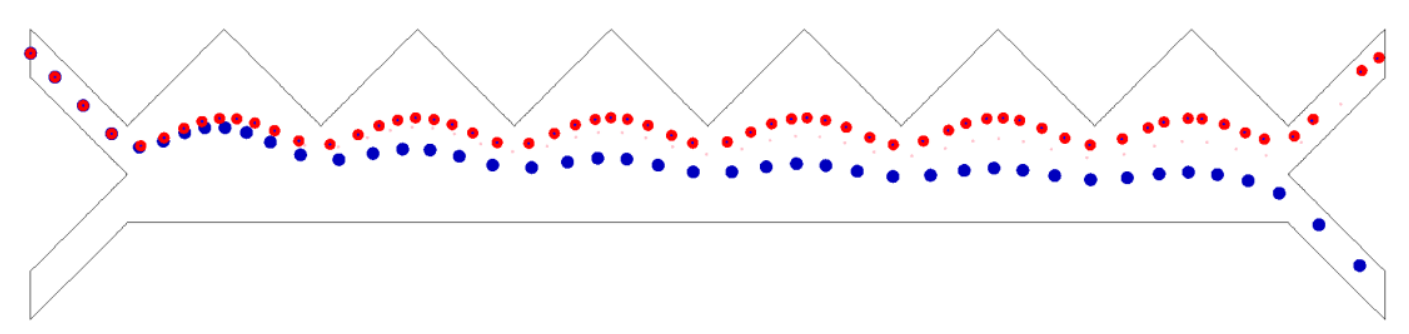

- The trajectory of cells without DEP

- The trajectory of CTC with DEP

The trajectory of erythrocyte with DEP

Figure 6. The trajectories of cells in channel when $u_{s}=200 \mu \mathrm{m} / \mathrm{s}, u_{b}=400 \mu \mathrm{m} / \mathrm{s}, f=$ 
$100 \mathrm{kHz}$, and $U_{0}=1.8 \mathrm{~V}$.

The separation efficiency of HOP-62 under different operation conditions (including the flowrate ratio of buffer and sample, and the electric potential of positive electrodes $U_{0}$ ) is shown in Fig. 7, where it can be seen that the separation of CTC and erythrocytes can be achieved when $1.9 \leq u_{b} / u_{s} \leq 2.5,1.6 \mathrm{~V} \leq U_{0} \leq 2.2 \mathrm{~V}$, and the sorting efficiency is able to reach approximately $99 \%$.
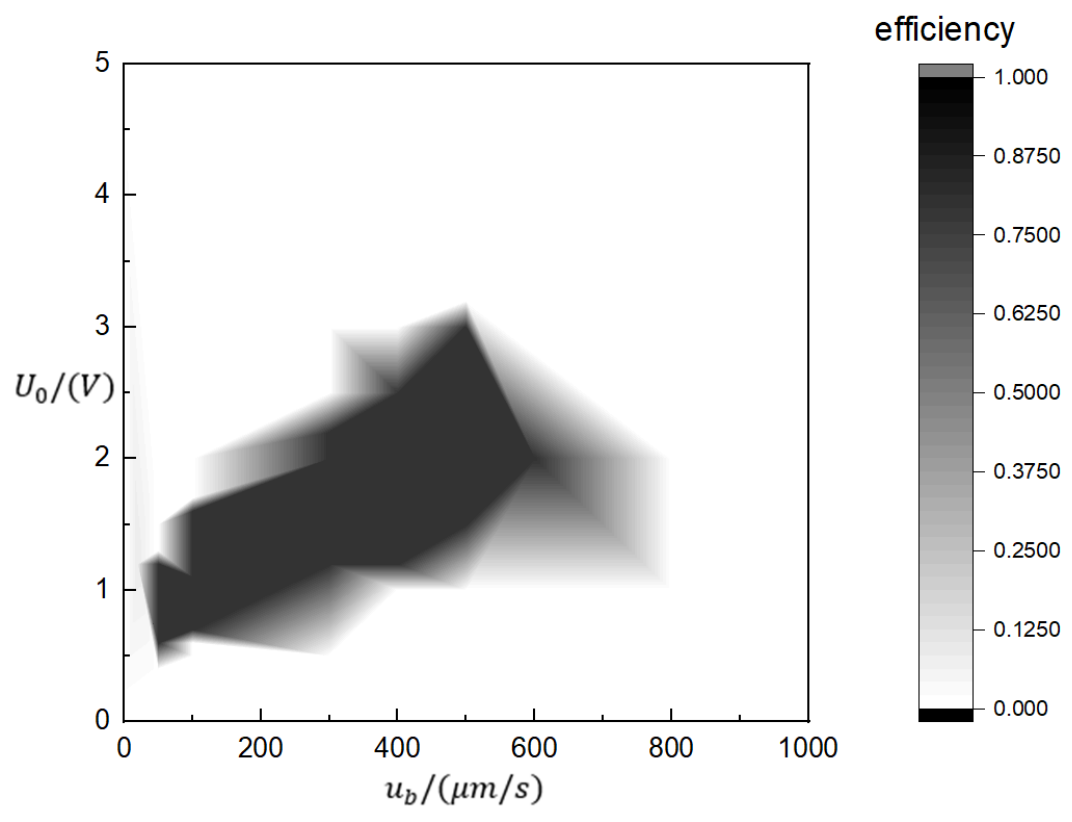

Figure 7. The separation efficiency of HOP-62 in DEP-assisted microfluidic device under different $u_{b}$ and $U_{0}, u_{s}=200 \mu \mathrm{m} / \mathrm{s}, f=100 \mathrm{kHz}$.

\section{Conclusions}

A novel DEP-based microfluidic strategy was presented in the paper where the cell sorting system is composed of low voltage electrodes and a microchannel in a "Y-Y" configuration. The mixture of erythrocytes and CTCs are fed into the flow channel from the upper inlet, whilst a carrying fluid is fed from the lower inlet to focus the cells to accumulate along upper side of the channel which features with successive triangles connected with 
successive alternatively reversed electrodes to induce a non-uniform electric field. When the mixture of cells flows through the long channel, they are subjected to the non-uniform electric field, inducing DEP forces with different magnitudes. Due to the size difference between CTCs and the erythrocytes, DEP force repels CTCs which will be deflected and exit through the lower outlet to achieve effective separation from other cells. The optimal operation conditions were investigated, and high separation efficiency can be obtained with $100 \mathrm{kHz} \mathrm{AC}$ when electrical potential from $1.6 \mathrm{~V}$ to $2.2 \mathrm{~V}$, and inlet flow velocity ratio from 1.9 to 2.5 are applied.

\section{Conflicts of interest}

The authors declare that they have no known competing financial interests or personal relationships that could have appeared to influence the work reported in this paper.

\section{Acknowledgements}

This work was financially supported by National Key R\&D Program of China under Grant No. 2018YFC0910601, National Natural Science Foundation of China under Grant No. 31971292 and 51981240009, Zhejiang Provincial Natural Science Foundation of China under Grant No. LY19E060001 and LQ19F050003, Ningbo Science and Technology Bureau under Service Industry Science \&Technology Programme with project code 2019F1030, as well as EU ThermaSMART project under Grant No. H2020-MSCA-RISE (778104)-Smart thermal management of high power microprocessors using phase-change (ThermaSMART). X.W. Xu acknowledges the financial support from the Doctoral 
Training Program between Ningbo Institute of Materials Technology and Engineering,

Chinese Academy of Sciences and University of Nottingham Ningbo China.

\section{References}

[1] R. A. Weinberg and R. A. Weinberg, The biology of cancer. Garland science, 2013.

[2] L. A. Liotta, J. Kleinerman, and G. M. Saidel, "Quantitative relationships of intravascular tumor cells, tumor vessels, and pulmonary metastases following tumor implantation," Cancer Res., vol. 34, no. 5, pp. 997-1004, 1974.

[3] M. Antfolk and T. Laurell, "Continuous flow microfluidic separation and processing of rare cells and bioparticles found in blood-A review," Anal. Chim. Acta, vol. 965, pp. 9-35, 2017.

[4] D. L. Adams et al., "Cytometric characterization of circulating tumor cells captured by microfiltration and their correlation to the cellsearch ${ }^{\circledR}$ CTC test," Cytom. Part A, vol. 87, no. 2, pp. 137-144, 2015.

[5] Y.-T. Lu et al., "NanoVelcro Chip for CTC enumeration in prostate cancer patients," Methods, vol. 64, no. 2, pp. 144-152, 2013.

[6] R. Königsberg et al., "Detection of EpCAM positive and negative circulating tumor cells in metastatic breast cancer patients," Acta Oncol. (Madr)., vol. 50, no. 5, pp. 700-710, 2011.

[7] A.-E. Saliba et al., "Microfluidic sorting and multimodal typing of cancer cells in self-assembled magnetic arrays," Proc. Natl. Acad. Sci., vol. 107, no. 33, pp. 14524-14529, 2010.

[8] B. Kwak et al., "Selective isolation of magnetic nanoparticle-mediated heterogeneity subpopulation of circulating tumor cells using magnetic gradient based microfluidic system," Biosens. Bioelectron., vol. 88, pp. 153-158, 2017.

[9] M. Hosokawa et al., "Size-based isolation of circulating tumor cells in lung cancer patients using a microcavity array system," PLoS One, vol. 8, no. 6, pp. e67466e67466, Jun. 2013, doi: 10.1371/journal.pone.0067466.

[10] P. B. Santhosh and N. P. Ulrih, "Multifunctional superparamagnetic iron oxide nanoparticles: promising tools in cancer theranostics," Cancer Lett., vol. 336, no. 1, pp. 8-17, 2013.

[11] H. A. Pohl, "The motion and precipitation of suspensoids in divergent electric fields," J. Appl. Phys., vol. 22, no. 7, pp. 869-871, 1951.

[12] J. Marchalot, J.-F. Chateaux, M. Faivre, H. C. Mertani, R. Ferrigno, and A.-L. Deman, "Dielectrophoretic capture of low abundance cell population using thick electrodes," Biomicrofluidics, vol. 9, no. 5, p. 54104, 2015.

[13] L. M. Broche, F. H. Labeed, and M. P. Hughes, "Extraction of dielectric properties of multiple populations from dielectrophoretic collection spectrum data," Phys. Med. Biol., vol. 50, no. 10, p. 2267, 2005.

[14] F. F. Becker, X.-B. Wang, Y. Huang, R. Pethig, J. Vykoukal, and P. R. Gascoyne, "Separation of human breast cancer cells from blood by differential dielectric affinity," Proc. Natl. Acad. Sci., vol. 92, no. 3, pp. 860-864, 1995. 
[15] J. Cheng, E. L. Sheldon, L. Wu, M. J. Heller, and J. P. O'Connell, "Isolation of cultured cervical carcinoma cells mixed with peripheral blood cells on a bioelectronic chip," Anal. Chem., vol. 70, no. 11, pp. 2321-2326, 1998.

[16] Y. Huang, S. Joo, M. Duhon, M. Heller, B. Wallace, and X. Xu, "Dielectrophoretic cell separation and gene expression profiling on microelectronic chip arrays," Anal. Chem., vol. 74, no. 14, pp. 3362-3371, 2002.

[17] R. Gambari et al., "Applications to cancer research of 'lab-on-a-chip' devices based on dielectrophoresis (DEP)," Technol. Cancer Res. Treat., vol. 2, no. 1, pp. 31-39, 2003.

[18] L. Altomare et al., "Levitation and movement of human tumor cells using a printed circuit board device based on software - controlled dielectrophoresis," Biotechnol. Bioeng., vol. 82, no. 4, pp. 474-479, 2003.

[19] P. R. C. Gascoyne, J. Noshari, T. J. Anderson, and F. F. Becker, "Isolation of rare cells from cell mixtures by dielectrophoresis," Electrophoresis, vol. 30, no. 8, pp. 1388-1398, 2009.

[20] T. B. Jones, "Basic theory of dielectrophoresis and electrorotation," IEEE Eng. Med. Biol. Mag., vol. 22, no. 6, pp. 33-42, 2003.

[21] H. A. Pohl, Dielectrophoresis: The behavior of neutral matter in nonuniform electric fields (Cambridge Monographs on physics). Cambridge/New York: Cambridge University Press, 1978.

[22] J. Rotow and T. G. Bivona, "Understanding and targeting resistance mechanisms in NSCLC," Nat. Rev. Cancer, vol. 17, no. 11, p. 637, 2017.

[23] M. R. King et al., "A physical sciences network characterization of circulating tumor cell aggregate transport," Am. J. Physiol. Physiol., vol. 308, no. 10, pp. C792-C802, 2015.

[24] P. R. C. Gascoyne, S. Shim, J. Noshari, F. F. Becker, and K. Stemke - Hale, "Correlations between the dielectric properties and exterior morphology of cells revealed by dielectrophoretic field - flow fractionation," Electrophoresis, vol. 34, no. 7, pp. 1042-1050, 2013.

[25] P. Fischer, "Understanding Viscoelasticity-An Introduction to Rheology (Nhan Phan-Thien)," Appl. Rheol., vol. 23, no. 6, p. 329, 2013. 